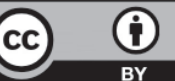

https://creativecommons.org/licenses/by/4.0/

\title{
MÉTODOS PARA IDENTIFICACIÓN DE PELIGROS, ANÁLISIS, EVALUACIÓN Y TRATAMIENTO DE LOS RIESGOS EN COLOMBIA
}

\author{
Methods for hazards identification, analysis, \\ evaluation and treatment of risks in Colombia
}

CARLOS ALIRIO BELTRÁN RODRÍGUEZ* JAIME ALEXANDER MURCIA PAMPLONA**

Recibido: 16 de junio de 2016. Aceptado: 27 de junio de 2016

DOI: http://dx.doi.org/10.21017/rimci.2016.v3.n6.a12

\section{Resumen}

El presente artículo presenta un análisis general de algunos modelos de identificación de peligros, análisis, evaluación y tratamiento de los riesgos en especial los de tipo laboral, los cuales forman parte de las metodologías de gestión integral del riesgo como uno de sus componentes principales. Algunas de las metodologías más reconocidas para desarrollar la gestión del riesgo en nuestro país, provienen de la integración de conceptos y estrategias implementadas por otros países u organizaciones no gubernamentales, las cuales han sido adaptadas de acuerdo a las características organizacionales de nuestra cultura laboral colombiana; por ello, en el presente estudio se muestra un análisis general de comparación por contenido de estas metodologías, las cuales permiten proponer un modelo integrado acorde a los modelos existentes ya utilizados en nuestras empresas y utilizados en diferentes sectores económicos; lo cual pretende facilitar su posible implementación de forma más eficiente, en especial para la prevención de la accidentalidad laboral desde la gestión del riesgo como una de sus principales estrategias.
\end{abstract}

Palabras clave: gestión del riesgo, métodos para identificación peligros, análisis, evaluación y tratamiento de los riesgos, modelos de evaluación de riesgos.

\begin{abstract}
This article presents an overview of some models of hazard identification, analysis, evaluation and treatment of risks especially in the workplace, as part of the methodologies of integrated risk management as one of its main components. Some of the most recognized methodologies to develop risk management in our country, come from the integration of concepts and strategies implemented by other countries or organizations non-governmental, which have been adapted according to organizational characteristics of our work culture Colombian; therefore, in this study a general comparison analysis is presented for the content of these methodologies, which allow proposing an integrated according to the
\end{abstract}

* Ingeniero Industrial de la Universidad Distrital Francisco José de caldas, de Bogotá, Colombia. Magister en Ingeniería Industrial, Especialista en Higiene y salud ocupacional de la Universidad Distrital Francisco José de caldas, de Bogotá, Colombia. Actualmente se desempeña como profesor de la Maestría de Ingeniería Industrial en el área de Salud Ocupacional y revisor de trabajos de grado. En pregrado en Ingeniería Industrial como docente en las áreas de Diseño y Materiales, Seguridad Industrial, Ergonomía, Métodos y Tiempos y Control de Calidad en la Universidad Distrital Francisco José de caldas en Bogotá, Colombia y es docente investigador del grupo de investigación Sistemas expertos y Simulación, Universidad Distrital (SES). Correo electrónico: cbeltran5@yahoo.es

** Ingeniero Industrial de la Universidad Distrital Francisco José de caldas, de Bogotá, Colombia Magister en Ingeniería Industrial, Especialista en Higiene y salud ocupacional de la Universidad Distrital Francisco José de caldas, de Bogotá, Colombia y Especialista en Ingeniería de Producción. Actualmente se desempeña como consultor en el área de la seguridad y la salud en el trabajo. Correo electrónico: ing_jaimemurcia@yahoo.com

Rev. Ingeniería, Matemáticas y Ciencias de la Información

Vol. 3 / Núm. 6 / julio - diciembre de 2016; pág. 29-38 
mode- existing model already used in our businesses and used in different economic sectors; which aims to facilitate possible implementation of more efficient, especially for the prevention of work-related injuries from risk management as one of its main strategies.

Keywords: risk management methods for hazard identification, analysis, evaluation and treatment of risks, risk assessment models.

\section{INTRODUCCIÓN}

En Colombia el Sistema General de Seguridad Social Integral se enfoca en la prevención y promoción de la salud, y el Sistema General de Riesgos Laborales hace énfasis en la protección del trabajador; de tal forma que éste cuente con condiciones dignas, justas y en un ambiente laboral donde se prevenga cualquier condición que represente peligro para su salud, ya sea por la manifestación de un accidente de trabajo o enfermedad laboral.

Desde el nivel estratégico de las organizaciones, es necesario plantear diferentes alternativas para gestionar los riesgos indistintamente de su campo de acción, tema o especificidad; de forma tal, que la empresa pueda establecer los parámetros necesarios que contengan diferentes elementos para su administración integral, desde la planificación, identificación, análisis, evaluación, tratamiento y seguimiento; de esta manera se define la gestión del riesgo como las actividades coordinadas para dirigir y controlar una organización con respecto al riesgo.

\section{Modelos DE GeSTión INTEGRAL DEL RIESGO}

Teniendo como punto de referencia los modelos generales para la gestión del riesgo referenciados en el marco teórico como son: Gestión del Riesgo NTC-ISO 31000:2011 [1], y la Guía Técnica Colombiana GTC-ISO 137 de 2011 [2], Modelo de Gestión del Riesgo Instituto Nacional de Seguridad e Higiene en el Trabajo (INSHT) [3], Gestión de Riesgo según Cortés [4] y Modelo de Gestión de Riesgo según Kerzner, H. (2003) [5], se realizó el siguiente análisis comparativo para establecer las características principales de cada modelo y contextualizar el enfoque del presente estudio de métodos para identificación de peligros, análisis, evaluación, tratamiento y monitoreo de los riesgos, y así realizar una propuesta de modelo para el sector laboral colombiano.

\section{GeSTIÓN DEL RIESGo NTC-ISO 31000:2011}

\section{A. Objetivo}

Establecer los principios y las directrices genéricas sobre la Gestión del Riesgo.

\section{B. Alcance}

Esta norma puede ser utilizada por cualquier empresa pública, privada o comunitaria, asociación, grupo o individuo; por lo tanto, no es específica para ninguna industria o sector, se puede aplicar a todos los niveles de una organización y a un amplio rango de actividades, incluyendo estrategias, decisiones, operaciones, actividades, peligros, riesgos laborales, procesos, funciones, proyectos, productos, servicios y activos. Esta norma se puede aplicar a cualquier tipo de riesgo, sin limitarse a su naturaleza, o si las consecuencias son positivas o negativas.

\section{Metodología}

El modelo ayuda a la gestión eficaz del riesgo, a través de la aplicación del proceso para la gestión del riesgo en diversos niveles y en contextos específicos de la organización. El marco garantiza que la información acerca del riesgo se deriva del proceso para la misma gestión del riesgo, se reporte de manera adecuada y se utilice como base para la toma de decisiones y la rendición de cuentas en todos los niveles pertinentes de la organización [6] (Fig. 1).

\section{MODELO DE GESTIÓN DEL RIESGO InSTITUTO Nacional DE SEgURIDAD e Higiene en el Trabajo (INSHT)}

\section{A. Objetivo}

Establecer los lineamientos generales necesarios para la Gestión del Riesgo.

Rev. Ingeniería, Matemáticas y Ciencias de la Información Vol. 3 / Núm. 6 / julio - diciembre de 2016; pág. 29-38 


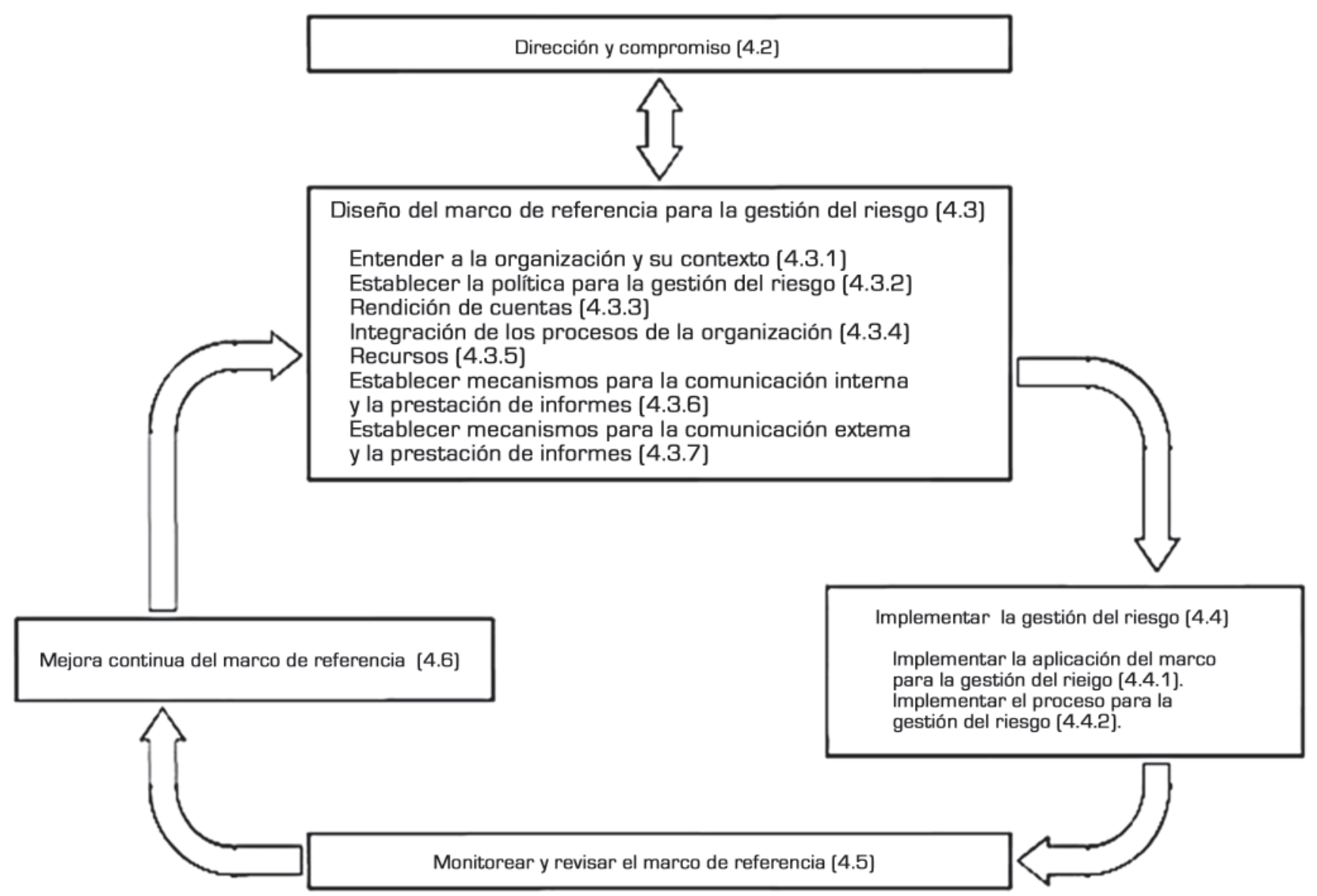

Fig. 1. Relación entre los componentes del marco de referencia para la gestión del riesgo. Fuente. Norma Técnica Colombiana NTC-ISO 31000 Gestión del Riesgo. Principios Directrices.

\section{B. Alcance}

El Instituto Nacional de Seguridad e Higiene en el Trabajo INSHT, establece a través de esta metodología, los parámetros y directrices generales para que puedan ser utilizados en diferentes sectores económicos y productivos; sin embargo tiene su mayor aporte en el sector laboral.

\section{Metodología}

El INSHT contempla para el modelo de Gestión del Riesgo el desarrollo sistémico de los procesos que involucran a todo nivel la evaluación y tratamiento de los riesgos, que a su vez requieren de identificación, análisis y valoración de los riesgos mediante elementos cualitativos y cuantitativos [3] (Fig. 2).

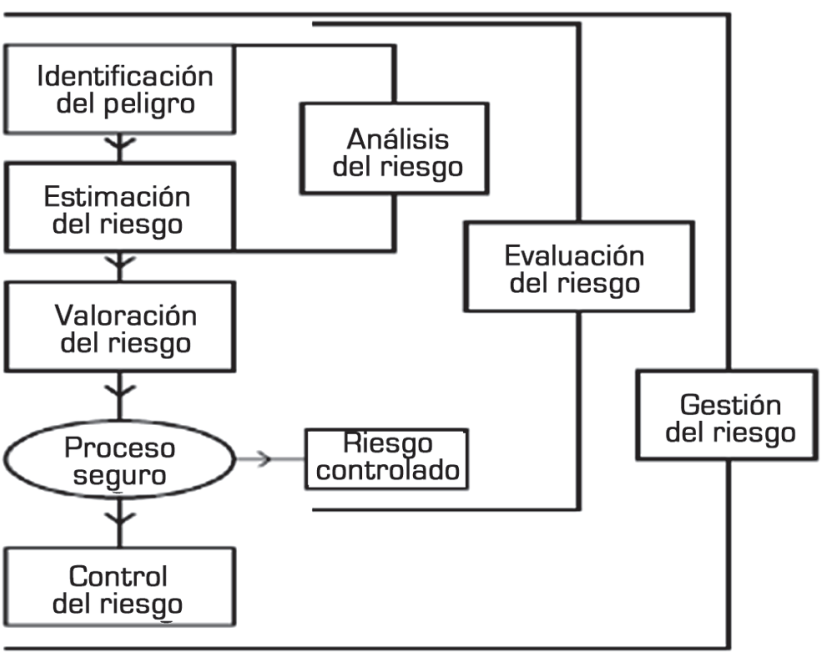

Fig. 2. Modelo Gestión del Riesgo Instituto Nacional de Seguridad e Higiene en el Trabajo [INSHT]. Fuente: Instituto Nacional de Seguridad e Higiene en el Trabajo [INSHT]. 


\section{MODELO DE GESTIÓN DEL RIESGO Cortés, J.M. ED. TÉbAR Flores. MADRID}

\section{A. Objetivo}

Proporcionar los principios generales para el análisis y valoración de riesgos necesarios para la gestión del riesgo.

\section{B. Alcance}

Tiene como fundamento la aplicación en el campo laboral; sin embargo cuenta con elementos comunes a otros modelos de gestión de riesgo, que pueden ser aplicados a otros campos productivos.

\section{Metodología}

El autor JM Cortés plantea que la gestión del riesgo esta sintetizada en dos etapas: el análisis del riesgo, que comprende las fases de identificación de peligros y estimación de los riegos, y la etapa de valoración del riesgo, que según él permite enjuiciar si los riesgos detectados resultan tolerables y en caso de no ser aceptables, deberán implementarse controles por la organización [4] (Fig. 3).

\section{MODELO DE GESTIÓN DEL RIESGO- Kerzner, H. Ed. Wiley. NeW Jersey}

\section{A. Objetivo}

Determinar las estrategias y métodos para la identificación y valoración de situaciones de riesgo.

\section{B. Alcance}

Establece métodos organizados y comprensivos para ser utilizados en la gestión, desde la planificación hasta el manejo, control y seguimiento del riesgo en diferentes campos de aplicación como son el laboral, financiero, público, económico, entre otros.

\section{Metodología}

Kerzner H. establece como fundamento y punto de partida la planificación desde el nivel estratégico de las organizaciones, en el cual se debe plantear diferentes alternativas para gestionar los riesgos, teniendo en cuenta igualmente la identificación, análisis de situaciones de riesgo, su evaluación, manejo y seguimiento de las medidas de control [5] (Fig. 4).

\section{AnÁlisis COMPARATIVO POR CONTE- NIDO DE METODOLOGÍAS DE GESTIÓN INTEGRAL DEL RIESGO}

Con el fin de establecer una evidencia objetiva acerca de las características individuales de las metodologías descritas anteriormente para la Gestión Integral de los Riesgos, así como las ventajas y desventajas de cada uno de los modelos, se presenta la siguiente Matriz de análisis comparativo por contenido de las metodologías de gestión integral del riesgo citadas anteriormente, la cual ha sido adaptada por el autor para el presente artículo de investigación (Fig. 5).

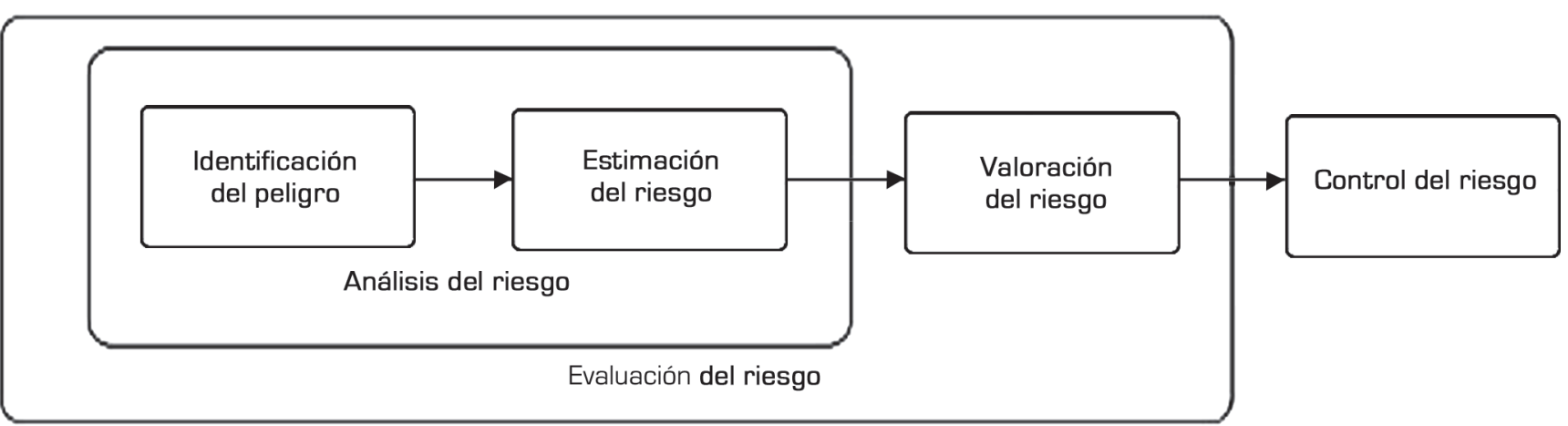

Fig. 3. Modelo de Gestión de Riesgo según Cortés. Fuente: Instituto Nacional de Seguridad e Higiene en el Trabajo [INSHT], http:/ / www.mtas.es/insht. 


\begin{tabular}{|c|c|c|c|c|}
\hline \multirow{2}{*}{$\begin{array}{c}\text { Planificación } \\
\text { del riesgo }\end{array}$} & $\begin{array}{c}\text { Identificación } \\
\text { del riesgo }\end{array}$ & $\begin{array}{c}\text { Análisis } \\
\text { del riesgo }\end{array}$ & $\begin{array}{c}\text { Manejo } \\
\text { del riesgo }\end{array}$ & Seguimiento \\
\cline { 2 - 3 } & Evaluación del riesgo & \\
\hline \multicolumn{4}{|c|}{ Gestión } \\
\hline
\end{tabular}

Fig. 4. Modelo de Gestión de Riesgo según Kerzner, H.

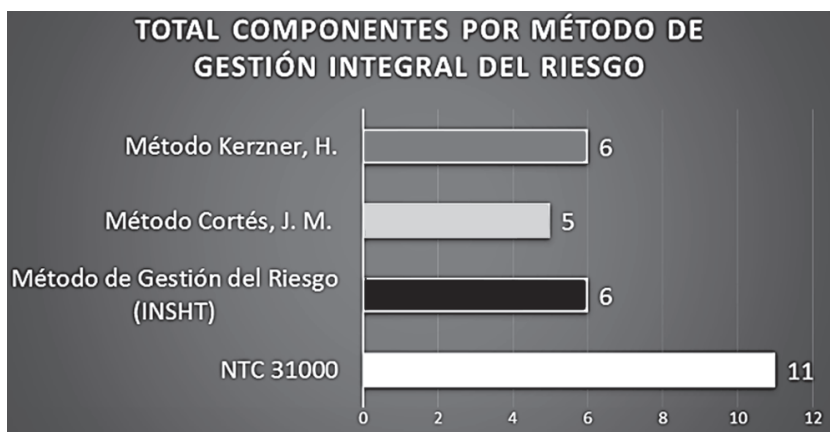

Fig. 5. Gráfica Componentes por método de gestión integral del riesgo. Fuente: Tesis de Investigación Diseño de Modelo de Gestión para el Control de las Variables que Intervienen en la Accidentalidad por Trabajos en Alturas, para los Obreros de la Construcción Vertical en Sector Público en Bogotá D.C. U. Distrital. Murcia Jaime. 2016.

De acuerdo a los diferentes modelos de Gestión Integral del Riesgo analizados, el modelo de gestión integral del riesgo que más tiene componentes comunes es la NTC 31000 definida por el Instituto Colombiano de Normas Técnicas ICONTEC; sin embargo, se aprecia que no cuenta con el concepto claramente establecido de identificación de peligro, como sí se encuentra en otras normas y leyes de aplicación nacional. De igual forma está metodología se tomó como referente principal para la propuesta de la método que permita identificar los peligros, analizar, evaluar y tratar los riesgos que influyen en la aparición de accidentes de trabajo y enfermedades laborales.

\section{MÉTOdos PARA IDENTIFICACIÓN DE PELIGROS, ANÁLISIS, EVALUACIÓN Y TRATAMIENTO DE LOS RIESGOS}

En el campo laboral nacional e internacional existen varios métodos para realizar la valoración o evaluación de los riesgos, según como lo defina cada autor; sin embargo, en general coinciden en que este componente hace parte fundamental de la Gestión del Riesgo, y que deben existir unas etapas mínimas a tener en cuenta para establecer una escala de valor frente a su posible manifestación y consecuencias.

La evaluación de riesgos es una actividad que se ha convertido en una obligación legal, con el fin de ayudar a que los responsables de la seguridad laboral adopten decisiones adecuadas frente a los peligros existentes. La evaluación de riesgos constituye el punto de partida para una gestión activa de la seguridad y la salud en el trabajo, ya que a partir de la información obtenida con la evaluación, se pueden establecer líneas de actuaciones válidas y eficaces, tomar decisiones cerca de la necesidad de establecer o no acciones preventivas y fijar criterios de aceptación. Es el proceso dirigido a estimar la magnitud de aquellos riesgos que no hayan podido evitarse, obteniendo la información necesaria para poder tomar decisiones apropiadas sobre la necesidad de adoptar medidas preventivas, y en tal caso, sobre qué tipo de medidas deben adoptarse.

De acuerdo al autor Rubio, J.C. (2004) « Métodos de evaluación de riesgos laborales». Ed. Días de Santos. Madrid, existen innumerables procedimientos para la evaluación de los riesgos; desde los más simples que se basan en consideraciones subjetivas de los trabajadores, hasta procedimientos cuantitativos que utilizan métodos estadísticos para determinar frecuencias, cálculos de daños, etc., y que son de aplicación generalizada para la evaluación de todos los tipos de riesgos. Los métodos de evaluación se clasifican en simplificados y complejos [7] como se presenta en la tabla 1 resumen adaptada por el autor del presente artículo.

\section{COMPARAción POR CONTENIDO ENTRE LOS MÉTODOS PARA IDENTIFICACIÓN DE PELIGROS, ANÁLISIS Y EVALUACIÓN, TRATAMIENTO Y MONITOREO DE LOS RIESGOS}

Para establecer las ventajas, desventajas, componentes y caracterización de los métodos analizados de identificación de peligros, evaluación, valoración, tratamiento y monitoreo de los riesgos, se desarrolló una matriz de comparación por contenido, la cual permitió establecer los elementos necesarios para proponer un método de bajo costo y que requiera moderados conocimientos técnicos especializados para su utilización en el 
Tabla 1. Clasificación de métodos de evaluación de riesgos.

\begin{tabular}{|c|c|c|c|}
\hline Tipos de evaluación de riesgos & \multicolumn{3}{|c|}{ Métodos de evaluación de riesgos } \\
\hline $\begin{array}{l}\text { Evaluación de riesgos impuestos por } \\
\text { legislación específica }\end{array}$ & & & \\
\hline \multicolumn{4}{|l|}{$\begin{array}{l}\text { Evaluación de riesgos para los que no } \\
\text { existe legislación específica }\end{array}$} \\
\hline \multicolumn{4}{|l|}{ Evaluación general de riesgos } \\
\hline \multirow{8}{*}{$\begin{array}{l}\text { Evaluación de riesgos que precisa } \\
\text { métodos especializados de análisis }\end{array}$} & Métodos simplificados & $\begin{array}{l}\text { Valoración simple o } \\
\text { método A,B, C: } \\
\text { El método binario: } \\
\text { El método fine: }\end{array}$ & \\
\hline & \multirow{7}{*}{ Métodos complejos } & \multirow{4}{*}{ Métodos cualitativos } & Análisis histórico \\
\hline & & & $\begin{array}{l}\text { Análisis preliminar de } \\
\text { riesgos }\end{array}$ \\
\hline & & & $\begin{array}{l}\text { Análisis de riesgos y } \\
\text { operabilidad [HAZOP] }\end{array}$ \\
\hline & & & Método Delphi \\
\hline & & \multirow[t]{3}{*}{ 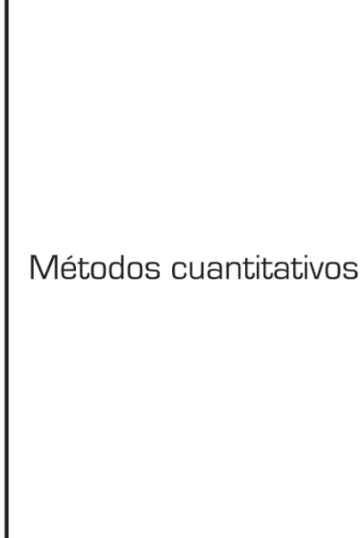 } & $\begin{array}{l}\text { Guía para la } \\
\text { identificación de } \\
\text { peligros y la valoración } \\
\text { de los riesgos en } \\
\text { Seguridad y Salud } \\
\text { Ocupacional - GTC } 45 \\
2012\end{array}$ \\
\hline & & & $\begin{array}{l}\text { Análisis de modos de } \\
\text { fallos y efectos (AMFE] }\end{array}$ \\
\hline & & & $\begin{array}{l}\text { Análisis del árbol de } \\
\text { fallos (FTA) }\end{array}$ \\
\hline
\end{tabular}

Fuente: Rubio, J.C. [2004] « Métodos de evaluación de riesgos laborales». Ed. Días de Santos, Madrid. Clasificación adaptada por el autor para trabajo de investigación.

campo laboral de cualquier tipo de organización; lo cual, a corto plazo es una ventaja para las empresas colombianas, que cuentan con procesos de diversas naturalezas, las cuales generan igualmente peligros y riesgos de distinta índole, y que pueden ser gestionados fácilmente con el método propuesto (tabla 2, Fig. 6).

De acuerdo a los diferentes componentes de los métodos para identificación de peligros, análisis y evaluación, tratamiento y monitoreo de los riesgos analizados, el Método que más tiene componentes comunes es la Guía para la Identificación de Peligros y la Valoración de los Riesgos en Seguridad y Salud Ocupacional - GTC 45 2012, definida por el

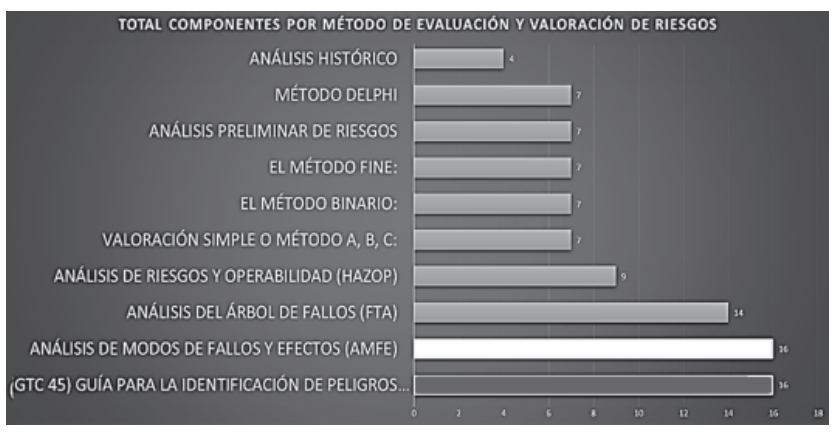

Fig. 6. Total componentes por método de evaluación y valoración de riesgos. Fuente: Tesis de Investigación Diseño de Modelo de Gestión para el Control de las Variables que Intervienen en la Accidentalidad por Trabajos en Alturas, para los Obreros de la Construcción Vertical en Sector Público en Bogotá D.C. U. Distrital. Murcia Jaime. 2016. Adaptado por el autor para trabajo de investigación. 
Tabla 2. Matriz de comparación por contenido de componentes de métodos para identificación de peligros, análisis y evaluación, tratamiento y monitoreo de los riesgos.

\begin{tabular}{|c|c|c|c|c|c|c|c|c|c|c|c|c|c|c|c|c|c|c|c|c|c|c|c|}
\hline \multicolumn{24}{|c|}{$\begin{array}{l}\text { Caracterización de componentes de métodos para identificación de peligros, análisis y evaluación, } \\
\text { tratamiento y monitoreo de los riesgos }\end{array}$} \\
\hline \multirow{3}{*}{\multicolumn{6}{|c|}{ Descripción y contexto }} & \multicolumn{13}{|c|}{ Valoración de riesgos NTC 31000} & \multirow{2}{*}{\multicolumn{5}{|c|}{\begin{tabular}{|l} 
Tratamiento/Acciones NTC 31000 \\
Tratamiento/Acciones NTC 31000
\end{tabular}}} \\
\hline & & & & & & $\begin{array}{c}\text { Iden. Pelig NTC } \\
31000\end{array}$ & \multicolumn{9}{|c|}{ Análisis de riesgos NTC 31000} & \multicolumn{3}{|c|}{$\begin{array}{l}\text { Evaluación de riesgos NTC } \\
\quad 31000\end{array}$} & & & & & \\
\hline & & & & & & $\begin{array}{c}\text { Iden. Pelig GTC } \\
45\end{array}$ & $\begin{array}{r}\text { An } \\
\text { riesc }\end{array}$ & álisis & de & & Eval & uació & $n$ de $\mathrm{r}$ & iesgo & s GTC & 45 & & $\begin{array}{l}\text { Valor riesgo } \\
\text { GTC } 45\end{array}$ & $\begin{array}{c}\text { Medidas de } \\
\text { Interv. GTC 45 }\end{array}$ & & & & \\
\hline 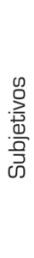 & 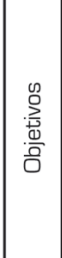 & 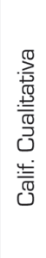 & 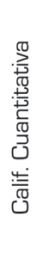 & 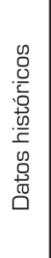 & 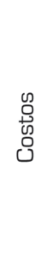 & 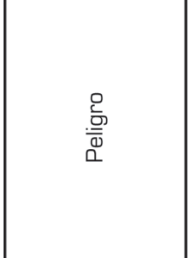 & 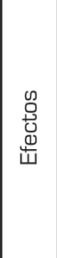 & 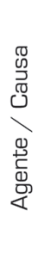 & 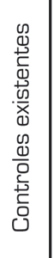 & $\begin{array}{l}\frac{0}{0} \\
\frac{0}{0} \\
\frac{0}{0} \\
\frac{0}{0} \\
\frac{c}{4}\end{array}$ & 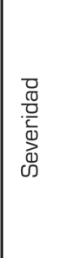 & 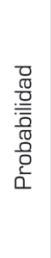 & $\begin{array}{l}\frac{.5}{0} \\
\frac{0}{0} \\
\frac{0}{x} \\
\frac{0}{4}\end{array}$ & 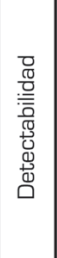 & 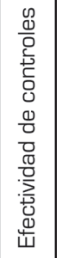 & 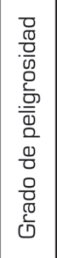 & 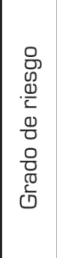 & 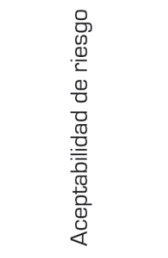 & 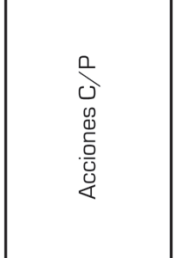 & $\begin{array}{l}\text { O } \\
\frac{\pi}{\square}\end{array}$ & 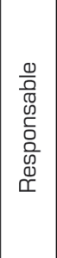 & 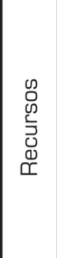 & 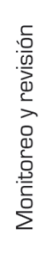 \\
\hline
\end{tabular}

Fuente: Tesis de Investigación Diseño de Modelo de Gestión para el Control de las Variables que Intervienen en la Accidentalidad por Trabajos en Alturas, para los Obreros de la Construcción Vertical en Sector Público en Bogotá D.C. U. Distrital. Murcia Jaime. 2016. Adaptado por el autor para trabajo de investigación.

Instituto Colombiano de Normas Técnicas ICONTEC [8] y el Análisis de Modos de Fallos y Efectos (AMFE) [9]; sin embargo, se aprecia que la primera no cuenta con elementos como objetivos en la descripción y contexto del análisis, análisis de costos, concepto de recursos, monitoreo y revisión claramente establecidos. Por otra parte, aunque el método AMFE es muy completo, es utilizado para procesos complejos con recursos técnicos especializados y por personal técnico calificado, lo que dificulta su implementación en la industria Colombiana; por lo tanto, se tomó la Guía para la Identificación de Peligros y la Valoración de los Riesgos en Seguridad y Salud Ocupacional - GTC 45 2012, como referente principal para la propuesta de la metodología que permita identificar los peligros, analizar, evaluar y tratar los riesgos que influyen en la aparición de accidentes y enfermedades laborales.

\section{Propuesta de método para IDENTIFICACIÓN DE PELIGROS, ANÁLISIS Y EVALUACIÓN, TRATAMIENTO Y MONITOREO DE LOS RIESGOS}

La siguiente propuesta de Método para la Identificación de Peligros, Análisis y Evaluación de los Riesgos, tratamiento y monitoreo, tiene en cuenta tres etapas que unifican las teorías y conceptos de los métodos estudiados [10], como son:

\section{A. Marco de referencia y contexto del riesgo}

El cual integra los aspectos organizacionales, estratégicos, administrativos y operativos de la actividad y tarea a analizar, los cuales son fundamentales para el adecuado enfoque del proceso de identificación del peligro y valoración del riesgo en general.

\section{B. Valoración de riesgos}

Se han encontrado diversas teorías en la literatura nacional e internacional, en donde no se cuenta con un criterio unificado acerca de la identificación de los peligros, análisis, evaluación y valoración de los riesgos [11].

\section{Tratamiento de los riesgos}

En esta etapa se propone incorporar criterios de formulación de controles basados en la etapa de valoración anterior, combinados con la definición de los tratamientos de los riesgos, formulación del Plan de Acción para la implementación de los controles propuestos en el tratamiento de los riesgos y Acciones de Seguimiento y Monitoreo de los mismos [12].

Por lo tanto, en este modelo se propone unificar las etapas y criterios para utilizar uniforme- 
mente los criterios anteriormente expuestos; de tal forma que una etapa general se denomine, Valoración de Riesgos, la cual contenga tres actividades: primero Identificación de Peligros, en la cual se referencia, describe y clasifica claramente el peligro a analizar, Segundo el Análisis de Riesgos cuyo propósito es el de establecer los agentes, efectos, y controles existentes del riesgo estudiado, Tercero la Evaluación de Riesgo: el cual pretende mediante un análisis cuali-cuantitativo dar valor al riesgo teniendo en cuenta los criterios ND Nivel de Deficiencia, NE Nivel de Exposición, NP Nivel de Probabilidad, INR Interpretación del nivel de Riesgo, NC Nivel de Consecuencia para finalmente determinar la AR aceptabilidad del Riesgo propuestos en la Guía para la Identificación de Peligros y la Valoración de los Riesgos en Seguridad y Salud Ocupacional - GTC 45 2012. A continuación a esta evaluación se establece si el riesgo es aceptable o no; en caso de ser aceptable, se pasa a la etapa siguiente del proceso, la cual es la determinación del tratamiento de los riesgos. En el caso que el riesgo no sea aceptable se propone como valor agregado al modelo, realizar un análisis adicional mediante el Concepto de Evaluación de Riesgo Residual, el cual está fundamentado en el control de los riesgos de la Gestión Pública (Dec. 943 de 2014, por el cual establece la Implementación de Modelo Estándar de Control Interno) [13], en el cual se debe realizar una valoración de la efectividad los controles existentes, y la formulación de otros controles adicionales con su valoración teórica esperada; de tal forma que, si el riesgo residual (adicional) en teoría es aceptado, se deben tener en cuenta estos controles adicionales en el tratamiento inicialmente propuesto durante el proceso de implementación.

De igual forma, se propone analizar y hacer seguimiento de forma continua durante la implementación de controles y tratamiento de los riesgos, así como a la efectividad de los mismos, y en caso de no encontrarse cumplimiento de los objetivos y metas propuestos, se deberá realizar nuevamente la Evaluación de Riesgo Residual; tal y como se describió anteriormente, lo que permitirá establecer si las medidas de tratamiento planteadas inicialmente fueron efectivas, son insuficientes, o si deben ajustarse para mejorar su funcionamiento.

Este modelo igualmente tiene en cuenta el ciclo PHVA, del que hace parte fundamental el proceso de Mejora Continua, en el cual para que exista una evolución de acuerdo a la visión de los evaluadores, debe tenerse en cuenta una retroalimentación proactiva, basada en el control y seguimiento permanente en cada una de las etapas; cuya evolución deberá verse reflejada en los ajustes de las entradas y procesos en cada inicio del ciclo PHVA, al igual que en la evaluación de los resultados, durante el seguimiento y monitoreo [14] (Fig. 7).

\section{Conclusiones}

Se han encontrado diversas teorías en la literatura nacional e internacional, en donde no se cuenta con un criterio unificado acerca de la identificación de los peligros, análisis, evaluación, valoración y tratamiento de los riesgos; es así como, mediante la propuesta de Método para Identificación de Peligros, Análisis y Evaluación, tratamiento y Monitoreo de los Riesgos, se pretende unificar estos criterios como alternativa de aplicación nacional, en diferentes campos de acción, en especial en el laboral.

En Colombia se evidencian varios conceptos básicos que difieren en su significado o estructura, los cuales han sido utilizados en varias normas legales o normas técnicas, que forman parte de los modelos más utilizados en nuestro país. Por lo tanto, es importante resaltar la necesidad de realizar una revisión y validación minuciosa de estos conceptos, su pertinencia y utilización en diversas publicaciones que son de dominio público, con el fin de establecer una uniformidad de los conceptos, y más aún en el campo de la gestión del riesgo [15].

Algunos modelos de gestión del riesgo utilizados en los aspectos administrativo, financiero, técnico, laboral, control interno (MECI), entre otros, han permitido la formulación del modelo planteado, ya que los componentes de la gestión del riesgo, en especial el de valoración de los riesgos, permite que desde el proceso de planificación, durante la valoración del riesgo establezca mecanismos para mejorar controles, más eficientes, enfocados a la prevención de la manifestación del riesgo, en esta caso los accidentes de trabajo y enfermedades laborales.

En la etapa de Tratamiento y monitoreo de los riesgos se propone incorporar criterios de formu-

Rev. Ingeniería, Matemáticas y Ciencias de la Información Vol. 3 / Núm. 6 / julio - diciembre de 2016; pág. 29-38 


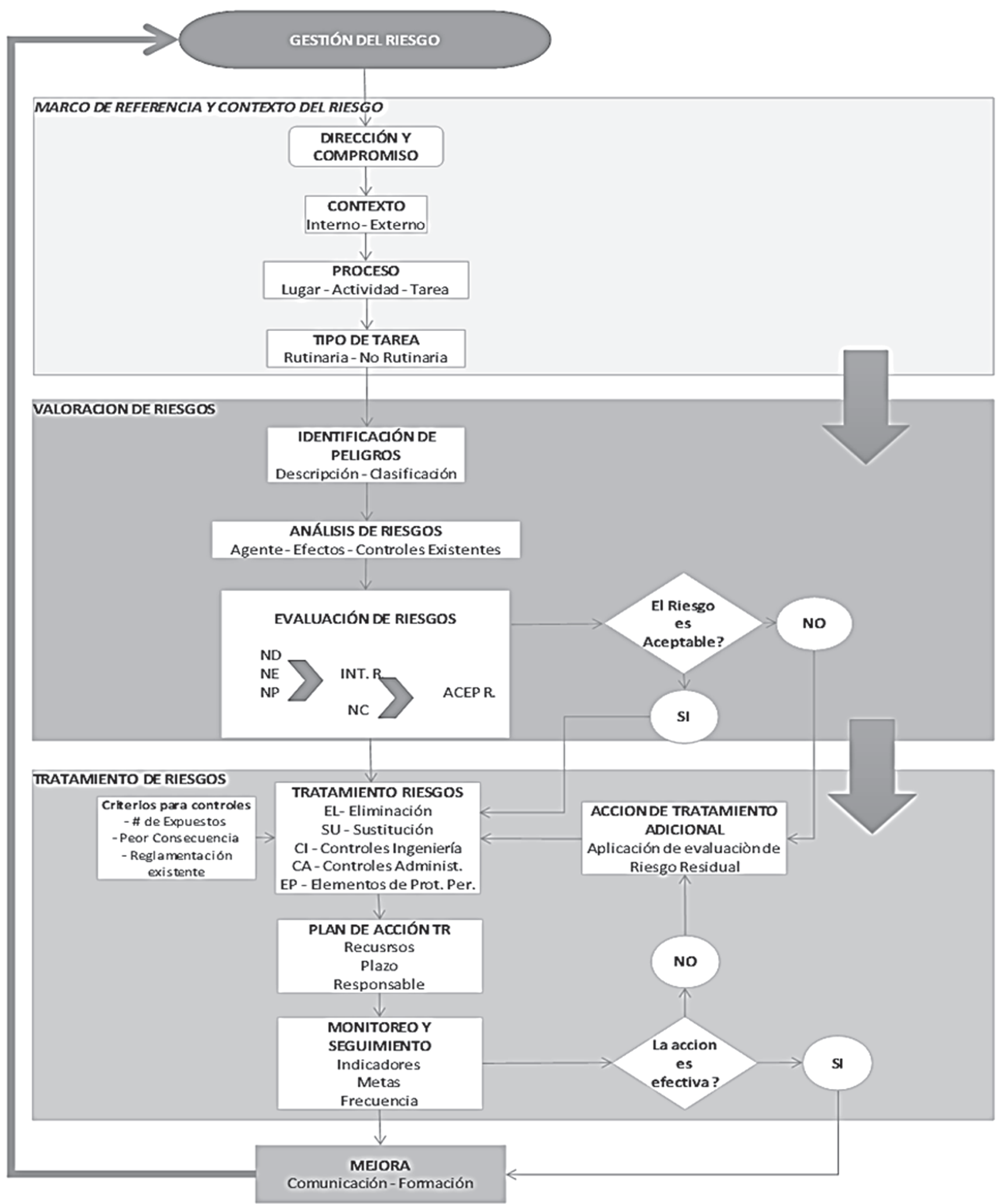

Fig. 7. Propuesta de método para identificación de peligros, análisis y evaluación, tratamiento y monitoreo de los riesgos. Fuente: Tesis de Investigación Diseño de Modelo de Gestión para el Control de las Variables que Intervienen en la Accidentalidad por Trabajos en Alturas, para los Obreros de la Construcción Vertical en Sector Público en Bogotá D.C. U. Distrital. Murcia Jaime. 2016. Adaptado por el autor para trabajo de investigación. 
lación de controles basados en la etapa de valoración, integrando la actividad de tratamiento de los riesgos, formulación del Plan de Acción para su Tratamiento y Acciones de Seguimiento y Monitoreo de los mismos en forma continua.

\section{REFERENCIAS}

[1] Guía Técnica Colombiana GTC-ISO 137 Gestión del riesgo. Vocabulario - Colombia. 2011.

[2] ICONTEC. Norma Técnica Colombiana NTC-ISO 31000 Gestión del Riesgo. Principios Directrices Colombia. 2011.

[3] INSHT. http://www.mtas.es/insht. Gráfico adaptado del Instituto Nacional de Seguridad e Higiene en el Trabajo (INSHT), el 12 de febrero de 2016.

[4] J. M. Cortés. «Técnicas de prevención de riesgos laborales». Ed. Tébar Flores, Madrid. 2000.

[5] H. Kerzner. Project management a systems approach to planning, scheduling and controlling». Ed. Wiley, New Jersey. 2003.

[6] H. Miller. ANSI Z359. Interpretación de la nueva Norma Z359-2007. [en línea]. USA. [Consultado el 01 de Noviembre de 2013].
[7] J. C. Rubio. « Métodos de evaluación de riesgos laborales». Ed. Días de Santos, Madrid. 2004.

[8] ICONTEC. Guía Técnica Colombiana GTC-ISO 45 Guía para la Identificación de Peligros y la Valoración de los Riesgos en Seguridad y Salud Ocupacional - Colombia. 2012.

[9] U. Hauptmanns. «Análisis de árboles de fallos». Ed. Bellaterra, Barcelona. 1986

[10] J. G. Martínez. Introducción al análisis de riesgos.

[11] ICONTEC. Norma Técnica Colombiana NTC-3701 - Higiene y Seguridad. Guía para la clasificación, registro y estadística de accidentes del trabajo y enfermedades profesionales - Colombia. 1995

[12] A. Creus. «Fiabilidad y seguridad - Su aplicación en procesos industriales».

[13] MINISTERIO DE TRABAJO. Decreto 943 de 2014. Implementación de Modelo Estándar de Control Interno, Departamento Administrativo de la Función Pública, Colombia.

[14] H. González, Consultor en Calidad y Gestión Empresarial, Buenos Aires, Argentina.

[15] ARSEG. Compendio de Normas Legales Sobre Salud Ocupacional. 2006. 